\title{
The Kinetic Burden of Potential Inversion in Two-Electron Electrochemical Reactions*
}

\author{
Dennis H. Evans \\ Department of Chemistry and Biochemistry, University of Delaware, Newark, DE 19716, USA \\ Dedicated to Professor Lennart Eberson on the occasion of \\ his 65th birthday
}

Evans, D. H., 1998. The Kinetic Burden of Potential Inversion in Two-Electron Electrochemical Reactions. - Acta Chem. Scand. 52: 194 197. () Acta Chemica Scandinavica 1998.

The electrochemical oxidation or reduction of many organic and organometallic compounds proceeds in a series of one-electron steps for which the addition or removal of the electron occurs with greater difficulty for a given step than for the one immediately preceding it. For example, for reduction of an aromatic hydrocarbon, $\mathrm{A}$, in an aprotic solvent, the addition of the first electron produces the corresponding radical anion and has associated with it a reversible formal potential, $E_{1}^{\circ \prime}$, standard heterogeneous electron-transfer rate constant, $k_{\mathrm{s} 1}$, and transfer coefficient, $\alpha_{1}$. In the second step, the radical anion accepts a second electron to form the dianion and the corresponding formal potential, $E_{2}^{\circ}$, is more negative than $E_{1}^{\circ}$. This is the normal ordering of potentials in

$$
\begin{aligned}
& \mathrm{A}+\mathrm{e}^{-}=\mathrm{A}^{-\cdot} ; \quad E_{1}^{\circ \prime}, k_{\mathrm{s} 1}, \alpha_{1} \\
& \mathrm{~A}^{-\cdot}+\mathrm{e}^{-}=\mathrm{A}^{2-} ; \quad E_{2}^{\circ \prime}, k_{\mathrm{s} 2}, \alpha_{2} \\
& 2 \mathrm{~A}^{-\cdot}=\mathrm{A}+\mathrm{A}^{2-}
\end{aligned}
$$

which introduction of the second electron occurs with greater difficulty than the first. For the purposes of this paper, we will limit consideration to two-electron reductions of neutral substrates. Transposition to oxidations and other charge types is easily accomplished.

\section{Results and discussion}

Procedures. The calculations presented in this paper were carried out using the simulation program DigiSim ${ }^{\mathbb{B}}$ (version 2.0, Bioanalytical Systems). An exponential factor of 0.5 was used for the expanded space grid and the step size was $1 \mathrm{mV}$. Planar diffusion was employed

\footnotetext{
* To whom correspondence should be addressed.
}

and in cases where the difference between anodic and cathodic peak potentials is reported, the switching potential was $-0.5 \mathrm{~V}$ with respect to $E_{\mathrm{ov}}^{\circ}$. In the simulations, the scan rate was $1 \mathrm{~V} \mathrm{~s}^{-1}, \alpha_{1}=\alpha_{2}=0.5$, all diffusion coefficients were $10^{-5} \mathrm{~cm}^{2} \mathrm{~s}^{-1}$ and $k_{\mathrm{s} 1}=k_{\mathrm{s} 2}$. Solution phase disproportionation [reaction (3)] of the intermediate was included with the rate constant set at $10^{9} 1 \mathrm{~mol}^{-1} \mathrm{~s}^{-1}$, close to the diffusion-controlled limit. The disproportionation reaction has little effect on the voltammograms except when the rate constant is small. As a cautionary note, it should be recognized that systems exhibiting potential inversion may involve large reorganization energies making the disproportionation reaction slow.

Reversible responses. Typically $E_{2}^{\circ \prime}$ falls several tenths of a volt negative of $E_{1}^{\circ}$. The principal underlying cause of this difference is the fact that more work is required to insert an electron into the radical anion than is required to add an electron to the neutral starting material. In fact, in the gas phase, $E_{1}^{\circ \prime}-E_{2}^{\supset \prime}$ is predicted to be $3-5 \mathrm{~V}$, its attenuation to a few tenths of a volt in solution being traceable to the effects of solvation.

In some instances, changes in structure occur upon reduction of $\mathrm{A}$ and these changes can cause modification of the relative energies of $\mathrm{A}, \mathrm{A}^{-}$and $\mathrm{A}^{2-}$ such that the two formal potentials become almost identical or even inverted, i.e., addition of the second electron can occur more easily than addition of the first. Such redox-induced structural changes are thought to be the underlying cause of potential inversion in all cases where it has been observed in the two-electron reactions of organic molecules. ${ }^{1}$

The extent of potential inversion can range from mild 
with $E_{1}^{\circ \prime}-E_{2}^{\circ \prime} \approx 0$ (a condition that might better be called potential compression) to quite strong with $E_{1}^{\circ \prime}-E_{2}^{\circ \prime}$ being as negative as $-0.2 \mathrm{~V}$. In this latter case voltammetric experiments (taking cyclic voltammetry as an example) will show no evidence of the individual electrontransfer reactions. Instead, a single reduction peak will be observed along with a single oxidation peak on the return sweep. In fact, if the electron transfers are facile (large $k_{\mathrm{s} 1}$ and $k_{\mathrm{s} 2}$ ) the voltammetric peak shape will be that of an overall two-electron reaction $\left(\Delta E_{\mathrm{p}}=\right.$ $E_{\mathrm{pa}}-E_{\mathrm{pc}} \rightarrow 57.0 / 2 \mathrm{mV}(298 \mathrm{~K})$ as $E_{1}^{\circ \prime}-E_{2}^{\circ \prime} \rightarrow-\infty ; E_{\mathrm{pa}}$ and $E_{\mathrm{pc}}$ are the anodic and cathodic peak potentials, respectively) and will be situated on the potential axis midway between the two formal potentials. Experimentally, one evaluates this apparent reversible potential for the overall two-electron process, $E_{\text {ov }}^{\circ}$, by finding the midpoint between the peak potentials as given in eqn. (4).

$E_{\mathrm{ov}}^{\circ \prime}=\frac{E_{\mathrm{pa}}+E_{\mathrm{pc}}}{2}=\frac{E_{1}^{\circ \prime}+E_{2}^{\circ \prime}}{2}$

This behavior is illustrated in Fig. 1, which shows the limiting shape and position of the reversible voltammogram (dashed curve) that would be observed for the case where $E_{1}^{\circ \prime}-E_{2}^{\circ \prime}$ is more negative than about $-0.2 \mathrm{~V}$. All of the above remarks are reiterations of well known facts about the properties of cyclic voltammograms. ${ }^{2-4}$

Sluggish electron-transfer kinetics. When small electrontransfer rate constants $\left(k_{\mathrm{s} 1}\right.$ and/or $\left.k_{\mathrm{s} 2}\right)$ are encountered, the voltammograms show features that resemble those seen with single electron-transfer reactions. The peaks become more drawn out along the potential axis (as determined by the magnitude of the transfer coefficients) and the difference between the peak potentials becomes larger. This behavior has also been treated in the literature with ample discussion of the effects of varying all of the kinetic parameters $\left(k_{\mathrm{s} 1}, \alpha_{1}, k_{\mathrm{s} 2}, \alpha_{2}\right) .^{5-7}$

The following comments serve to emphasize a point which is at least implicitly present in the earlier papers

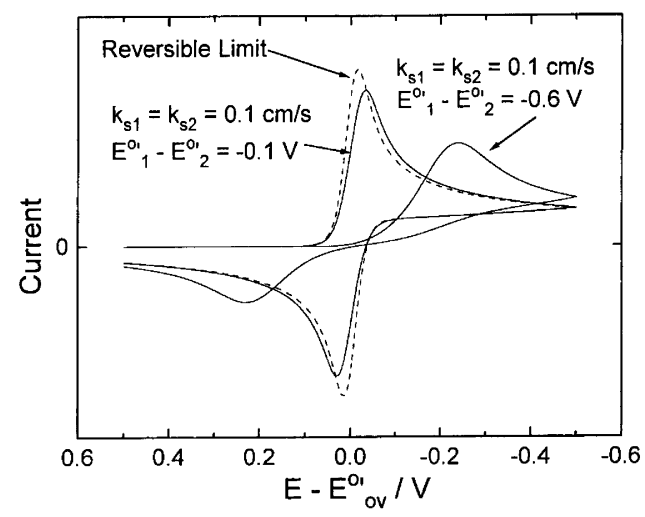

Fig. 1. Simulated voltammograms for two-electron reduction with potential inversion. Current scale is arbitrary. Dashed: reversible limit $\left(k_{\mathrm{s} 1}, k_{\mathrm{s} 2} \rightarrow \infty\right)$ with $E_{1}^{\prime \prime}-E_{2}^{\prime \prime}=-0.6 \mathrm{~V}$. Other voltammograms as labeled. but may not be immediately obvious to the reader. While carrying out numerous simulations on systems with inverted potentials, it was noted ${ }^{1}$ that abnormally large values of $k_{\mathrm{s} 1}$ and $k_{\mathrm{s} 2}$ were needed in order to produce voltammograms whose shape was close to reversible. Thus potential inversion causes the behavior to be less reversible compared with normal ordering of potentials. This effect will be termed the 'kinetic burden' of potential inversion.

We will not develop a general treatment in terms of dimensionless parameters and kinetic zone diagrams but, instead, the phenomenon will be illustrated by simulations using typical values of parameters and experimental conditions. In addition to the reversible voltammogram shown in Fig. 1 (dashed curve), two other voltammograms show the effect of the kinetic burden. First, the rate constants $\left(k_{\mathrm{s} 1}=k_{\mathrm{s} 2}=0.1 \mathrm{~cm} \mathrm{~s}^{-1}\right)$ and scan rate $\left(1 \mathrm{~V} \mathrm{~s}^{-1}\right)$ were set at values that would ordinarily give a quite reversible response ( $\Delta E_{\mathrm{p}}$ for a one-electron process would exceed the reversible limit by only $9 \mathrm{mV}$ ). However, even with $-0.1 \mathrm{~V}$ potential inversion, the cathodic and anodic peaks are beginning to separate, the value of $\Delta E_{\mathrm{p}}$ being $32 \mathrm{mV}$ larger than the reversible limit of $30 \mathrm{mV}$. When the potential inversion was increased to $-0.6 \mathrm{~V}$ while using the same rate constants, $\Delta E_{\mathrm{p}}$ became $0.47 \mathrm{~V}$, almost one-half volt greater than the reversible limit! Clearly, potential inversion will cause a system with given electron-transfer rate constants to behave less reversibly than it would with normal ordering of potentials.

The qualitative reasons underlying this behavior can be easily appreciated if one considers that the cathodic peak for the voltammogram with $-0.6 \mathrm{~V}$ potential inversion appears at $-0.24 \mathrm{~V}$ which is $60 \mathrm{mV}$ positive of $E_{1}^{\circ}$ $(-0.3 \mathrm{~V})$. Here the forward rate constant for insertion of the first electron, $k_{\mathrm{f} 1}$, (which is the rate-limiting step) is only about one-third of $k_{\mathrm{s} 1}$ and is just large enough at this potential to support the mass-transport controlled currents that must be achieved at and beyond the peak. Clearly, $k_{\mathrm{f} 1}$ would be much too small at potentials near $E_{\mathrm{ov}}^{\circ}$ to support mass-transport controlled currents. Thus the very negative value of $E_{1}^{\circ \prime}$ inevitably drags the cathodic peak to more negative potentials.

On the return scan the rate constant of the ratelimiting step is $k_{\mathrm{b} 2}$ and the very positive position of the anodic peak is explained by completely analogous arguments.

Of course, the magnitude of the kinetic burden will depend upon the rate constants, $k_{\mathrm{s} 1}$ and $k_{\mathrm{s} 2}$. This is illustrated in Fig. 2 where $\Delta E_{\mathrm{p}}-\Delta E_{\mathrm{p} . \text { rev }}$ is plotted for varying degrees of potential inversion and a number of different rate constants, assuming for illustration that $k_{\mathrm{s} 1}=k_{\mathrm{s} 2}$. Of course, as the rate constants become larger, a higher degree of inversion can be tolerated with retention of reversible behavior. In the limit of very large potential inversions where the system is behaving in a totally irreversible fashion, the slope of the $\Delta E_{\mathrm{p}}-\Delta E_{\mathrm{p} . \mathrm{rev}}$ vs. $E_{1}^{\circ \prime}-E_{2}^{\circ \prime}$ plots approaches unity. This is due to the fact that the irreversible cathodic peak depends directly on $E_{1}^{\circ \prime}$ and the irreversible anodic peak moves in concert 


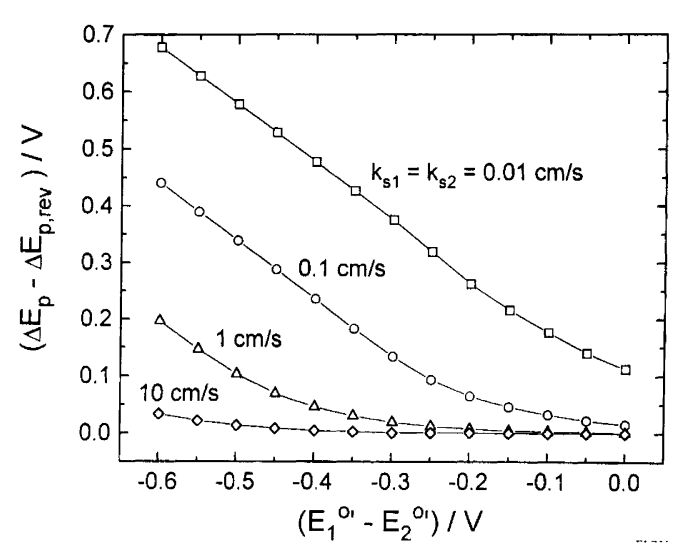

Fig. 2. Increase in peak separation beyond the reversible limit as a function of the extent of potential inversion.

with $E_{2}^{\circ} \cdot{ }^{2}$ It is interesting to note that even with rate constants at the maximum values normally encountered $\left(1-10 \mathrm{~cm} \mathrm{~s}^{-1}\right),{ }^{8}$ there can be significant deviations from reversible behavior when the potentials are inverted.

On the question of simultuneous is. stepwise mo-electron transfer. It is presently well accepted that two-electron reactions are composed of at least two distinct elementary steps. That is, even when two electrons are transferred at the same potential, the process involves successive transfer of single electrons. There are some authors, however, who are not willing to rule out the possibility of simultaneous transfer of a pair of electrons. ${ }^{9}$

Assuming that a stepwise mechanism holds, we may ask under what conditions is the lifetime of the intermediate $\mathrm{A}^{-\cdot}$ so short that it is not reasonable to consider it to be an actual intermediate in the process. When the potentials are inverted, $k_{\mathrm{f} 2}$ can be very large at the cathodic reduction peak and thus, once formed, $\mathrm{A}^{-\cdot}$ will be rapidly reduced to $\mathrm{A}^{2-}$. A reasonable definition of the point at which it is no longer reasonable to call $\mathrm{A}^{-}$. a true intermediate might be where $\mathrm{A}^{-}$is reduced before it is able to escape from the electrode surface.

The effective rate constant for diffusive escape of $\mathrm{A}^{-}$. is $D / d$, where $D$ is the diffusion coefficient of $\mathrm{A}^{-\cdot}$ and $d$ is the minimum distance that $\mathrm{A}^{-\cdot}$ must move before it is considered to have escaped from the electrode and become a freely diffusing intermediate.* Taking $D$ to be $10^{-5} \mathrm{~cm}^{2} \mathrm{~s}^{-1}$ and $d$ to be $10 \AA$ we obtain $100 \mathrm{~cm} \mathrm{~s}^{-1}$ for the effective rate constant for diffusive escape. Whenever $k_{\mathrm{f} 2}$ exceeds $100 \mathrm{~cm} \mathrm{~s}^{-1}$, it is more likely that $\mathrm{A}^{-\cdot}$ will be reduced to $\mathrm{A}^{2-}$ than diffuse away from the electrode surface.

* This formulation is developed by writing the linearized form of Fick's first law for diffusion of the intermediate: flux $=$ $-D(\Delta C / \Delta x)$. If the concentration of the intermediate at the electrode is $C$ and it diffuses through distance $d$ to a region where no intermediate exists, $\Delta C=-C$ and $\Delta x=d$. Thus, the diffusive flux away from the electrode is $D(C / d)$. By contrast, the reactive flux for the electron transfer is $k_{\mathrm{f} 2} C$. This is the justification for comparing $D / d$ with $k_{\mathrm{f} 2}$.
What conditions will lead to $k_{\mathrm{f} 2}>100 \mathrm{~cm} \mathrm{~s}^{-1}$ at $E_{\mathrm{pc}}$, for example, so that one could say that the reaction at this potential was effectively the simultaneous transfer of two electrons? If the reaction is totally irreversible $\left(E_{\mathrm{pc}}\right.$ more than about $200 \mathrm{mV}$ negative of its reversible value, $E_{\mathrm{pc} . \mathrm{rev}}$ ) it can be shown ${ }^{\dagger}$ that $k_{\mathrm{f} 2}$ at $E_{\mathrm{pc}}$ is given by eqn. (5) where $\alpha$ is the assumed common value of $\alpha_{1}$ and $\alpha_{2}, D$ is the diffusion coefficient of $\mathrm{A}, v$ is the scan rate

$\ln k_{\mathrm{f} 2}=\frac{\alpha E}{R T}\left(E_{2}-E_{1}\right)+0.780+\ln \sqrt{\frac{D \alpha F v}{R T}}$

and $k_{\mathrm{s} 1}$ is assumed to equal $k_{\mathrm{s} 2}$. For the conditions used in the calculations in this paper $(\alpha=0.5, D=$ $10^{-5} \mathrm{~cm}^{2} \mathrm{~s}^{-1}, v=1 \mathrm{~V} \mathrm{~s}^{-1}, T=298 \mathrm{~K}$ ), eqn. (5) requires $E_{1}^{\prime \prime}-E_{2}^{\circ \prime}$ to be equal or more negative than $-0.416 \mathrm{~V}$ in order for $k_{\mathrm{f} 2} \geqslant 100 \mathrm{~cm} \mathrm{~s}^{-1}$. This calculation shows that as long as totally irreversible voltammograms are obtained, the reduction process at the cathodic peak will effectively involve the simultaneous transfer of two electrons only for cases where strong potential inversion occurs.

When less severe inversion is encountered, it is still possible to have a large value of $k_{\mathrm{f} 2}$ as long as there are correspondingly large values of $k_{\mathrm{s}}$. For example, at $E_{1}^{\circ \prime}-E_{2}^{\circ \prime}=-0.3 \mathrm{~V}$, the common value of $k_{\mathrm{s}}$ must exceed $4 \mathrm{~cm} \mathrm{~s}^{-1}$ in order for $k_{\mathrm{f} 2}$ to be greater than $100 \mathrm{~cm} \mathrm{~s}^{-1}$ at $E_{\mathrm{pc}}$. For $E_{1}^{\circ \prime}-E_{2}^{\circ \prime}=-0.2 \mathrm{~V}$, the minimum $k_{\mathrm{s}}$ climbs to $11 \mathrm{~cm} \mathrm{~s}^{-1}$ and $k_{\mathrm{s}}$ greater than $28 \mathrm{~cm} \mathrm{~s}^{-1}$ is needed at $E_{1}^{\circ \prime}-E_{2}^{\circ \prime}=-0.1 \mathrm{~V}$. Thus it is unlikely that effectively simultaneous two-electron reduction will occur with modest potential inversion. In fact, as $10 \mathrm{~cm} \mathrm{~s}^{-1}$ is close to the maximum value of $k_{\mathrm{s}}$, which to the author's knowledge has been reported, potential inversions exceeding $200 \mathrm{mV}$ must be present in order to see the effectively one-step two-electron transfer. In cases where $E_{1}^{\circ \prime}-E_{2}^{\circ \prime}$ has been measured, it is usually between 0 and perhaps $-200 \mathrm{mV}$ at the maximum. Thus, it appears likely that all known cases of two-electron reactions with potential inversion ${ }^{1}$ actually occur by the conventional stepwise process in which it is meaningful to regard $\mathrm{A}^{-}$. (or its analogue) as a true intermediate.

It must be emphasized that the numerical conclusions that have been presented are based on a limited set of conditions: $\alpha_{1}=\alpha_{2}=0.5, k_{\mathrm{s} 1}=k_{\mathrm{s} 2}$, all diffusion coefficients equaling $10^{-5} \mathrm{~cm}^{2} \mathrm{~s}^{-1}, v=1 \mathrm{~V} \mathrm{~s}^{-1}, T=298 \mathrm{~K}$. However, these same issues can easily be addressed for any other conditions.

Conclusions. This brief paper comments upon and extends published theoretical work concerning electrontransfer kinetics in two-electron reactions with potential inversion. The discussion focuses on the fact that, for given values of the standard heterogeneous electron-

\footnotetext{
${ }^{\dagger}$ The first electron-transfer reaction is rate determining so the

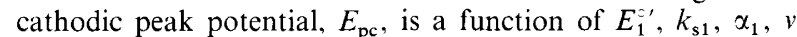
and $D$ and is given by eqn. (50), Ref. 2. $k_{\mathrm{f} 2}$ at $E_{\mathrm{pc}}$ is related to $E_{2}^{\circ}, k_{\mathrm{s} 2}, \alpha_{2}$ by the Butler-Volmer equation.
} 
transfer rate constants, potential inversion will bring about an apparent decrease in the reversibility of the reaction. This phenomenon has been termed the 'kinetic burden' of potential inversion.

A means to determine whether a two-electron reaction proceeds in a stepwise fashion by successive transfer of electrons or is effectively a simultaneous two-electron process has been proposed. The test is based on establishing whether the intermediate can escape from the electrode before reacting in the second step. Using this criterion, it is concluded that the effectively simultaneous two-electron reaction can only be seen when extensive potential inversion occurs, definitely more than $200 \mathrm{mV}$ inversion and more probably greater than $400 \mathrm{mV}$ where it has been shown that totally irreversible reactions will always occur by effectively simultaneous transfer of two electrons.
Acknowledgements. This research was supported by the National Science Foundation of the United States.

\section{References}

1. Evans, D. H. and Hu, K. J. Chem. Soc., Faraday Trans. 92 (1996) 3983.

2. Nicholson, R. S. and Shain, I. Anal. Chem. 36 (1964) 706.

3. Polcyn, D. S. and Shain, I. Anal. Chem. 38 (1966) 370.

4. Myers, R. L. and Shain, I. Anal. Chem. 41 (1969) 980.

5. Ruzic, I. J. Electroanal. Chem. 52 (1974) 331.

6. Ryan, M. D. J. Electrochem. Soc. 125 (1978) 547.

7. Hinkelmann, K. and Heinze, J. Ber. Bunsenges. Phys. Chem. 91 (1987) 243.

8. Fawcett, W. R. and Opallo, M. Angew. Chem., Int. Ed. Engl. 33 (1994) 2131.

9. Tributsch, H. J. Electroanal. Chem. 331 (1992) 783.

Received March 6, 1997. 